\title{
Diagnostic accuracy in detecting tears in the proximal biceps tendon using standard nonenhancing shoulder MRI
}

This article was published in the following Dove Press journal:

Open Access Journal of Sports Medicine

28 April 2014

Number of times this article has been viewed

\author{
Samuel A Dubrow' \\ Jonathan J Streit ${ }^{2}$ \\ Yousef Shishani² \\ Mark R Robbin ${ }^{3}$ \\ Reuben Gobezie ${ }^{2}$ \\ 'Department of Orthopedics, \\ Alegent Creighton Clinic, Creighton \\ University School of Medicine, \\ Omaha, NE, USA; ${ }^{2}$ Department of \\ Orthopedics, Cleveland Shoulder \\ Institute, ${ }^{3}$ Department of Radiology, \\ University Hospitals of Cleveland, \\ Cleveland, $\mathrm{OH}$, USA
}

Background: There is a paucity of data in the literature evaluating the performance of noncontrast MRI in the diagnosis of partial and complete tears of the proximal portion of the long head of the biceps (LHB) tendon. The objective of this study was to evaluate the accuracy of noncontrast magnetic resonance imaging (MRI) compared to arthroscopy for the diagnosis of pathology involving the intra-articular portion of the LHB tendon.

Methods: We conducted a retrospective review of 66 patients (mean age 57.8 years, range 43-70 years) who underwent shoulder arthroscopy and evaluation of the LHB tendon after having had a noncontrast MRI of the shoulder. Biceps pathology was classified by both MRI and direct arthroscopic visualization as either normal, partial tearing, or complete rupture, and arthroscopy was considered to be the gold standard. We then determined the sensitivity, specificity, and positive- and negative-predictive values of MRI for the detection of partial and complete LHB tears.

Results: MRI identified 29/66 (43.9\%) of patients as having a pathologic lesion of the LHB tendon (19 partial and ten complete tears) while diagnostic arthroscopy identified tears in $59 / 66$ patients $(89.4 \%$; 50 partial and 16 complete). The sensitivity and specificity of MRI for detecting partial tearing of the LHB were $27.7 \%$ and $84.2 \%$, respectively (positive predictive value $=81.2 \%$, negative predictive value $=32.0 \%$ ). The sensitivity and specificity of MRI for complete tears of the LHB were $56.3 \%$ and $98.0 \%$, respectively (positive predictive value $=90.0 \%$, negative predictive value $=87.5 \%$ )

Conclusion: Standard noncontrast MRI of the shoulder is limited in detecting partial tears and complete ruptures of the intra-articular LHB tendon. Surgeons may encounter pathologic lesions of the LHB tendon during arthroscopy that are not visualized on preoperative MRI.

Keywords: long head biceps tendon, biceps tendon tear, MRI detection, magnetic resonance imaging, case series

\section{Introduction}

The long head of the biceps (LHB) tendon is an important pain generator in the anterior shoulder, with both sensory and sympathetic innervation, which may transmit pain signals in response to inflammatory or degenerative tendinitis, instability, partial tears, or complete rupture. ${ }^{1-4}$ The diagnosis of LHB pathology by physical examination is difficult, as anterior shoulder pain may also be attributable to acromioclavicular joint pathology, subacromial impingement, coracoid impingement, or pathology of the rotator cuff. ${ }^{5}$ Imaging of the shoulder with plain radiography and magnetic resonance imaging (MRI) is often helpful in identifying shoulder pathology, but in many cases isolated LHB pathology remains a diagnosis of exclusion. The typical plain radiographic views (anteroposterior, lateral, axillary) for shoulder evaluation are seldom
Correspondence: Samuel A Dubrow Department of Orthopedics, Alegent Creighton Clinic, Creighton University School of Medicine, Lakeside ONE Professional Center, 16909 Lakeside Hills Court, Suite 300, Omaha, NE 68130, USA

$\mathrm{Tel}+\mathrm{I} 4027170820$

Fax +I 4027170830

Email samueldubrow@gmail.com 
helpful in diagnosing LHB tendinitis, tearing, and rupture. ${ }^{4}$ Other imaging techniques such as MRI with intravenous gadolinium, MR arthrography, and ultrasound have been studied, but the results have also not been favorable. ${ }^{6-10}$ As far as we are aware, there are no studies in the literature that have evaluated the performance of noncontrast MRI in the diagnosis of LHB pathology.

The purpose of this study was to evaluate the accuracy of noncontrast MRI in diagnosing pathology of the intraarticular portion of the LHB, using arthroscopic examination as the gold standard. We endeavored to calculate the sensitivity, specificity, positive predictive value (PPV), and negative predictive value (NPV) of MRI for the detection of both partial and complete LHB tears. Based on the results of previous studies evaluating advanced imaging, we hypothesized that standard noncontrast MRI would not be accurate in the detection of LHB pathology.

\section{Materials and methods}

We conducted a retrospective review of 66 patients (mean age 57.8 years, range 43-70 years; 24 female, 42 male) who received a noncontrast MRI of the shoulder and subsequently underwent shoulder arthroscopy between September 2007 and May 2011. The institutional review board of our hospital approved the design and protocol of this study. As a criterion for inclusion in the study, a diagnosis of LHB tendinitis, partial or complete LHB tear, LHB instability, or superior labrum anterior posterior tear must have been suspected based on physical examination. All patients were active individuals looking for relief of pain in their shoulders for continued participation in various athletic activities. All failed conservative treatments consisting of activity modification, physical therapy, and corticosteroid injections for a period of at least 4 months. Exclusion criteria included patients with previous surgery, fracture, inflammatory arthritis, or a diagnosis of adhesive capsulitis.

Our preoperative physical examination included the Speed's test, ${ }^{11}$ O'Brien's sign, ${ }^{12}$ and tenderness over the bicipital groove. All patients underwent a noncontrast MRI prior to surgery, which was reviewed by a fellowshiptrained musculoskeletal radiologist, with an average time of 1.55 months between MRI and arthroscopy. MRI was performed at a single institution using the same 3.0 Tesla GE machine (GE Healthcare, Piscataway, NJ, USA), with proton density, T1 and T2 sequences in three planes (axial, coronal, sagittal). An MRI diagnosis of a partial LHB tendon tear was defined as any qualitative interpretation by the radiologist as an intra-articular split, fraying, or partial rupture.
Absence of the LHB in the groove and distal displacement indicated a complete rupture or tear. An MRI diagnosis of a "non-tear" was defined as the radiologist's interpretation of a "normal biceps tendon" or "intact with changes consistent with degeneration, tendinopathy, or tenosynovitis."

The decision to proceed with surgery was made based upon physical examination findings as well as advanced imaging. In most cases, concomitant shoulder pathology was also suspected based upon physical examination and imaging, and the surgical plan included addressing all pathology within the shoulder at the time of arthroscopy. Preoperative diagnoses included rotator cuff tears, biceps tendinitis or tearing, superior labrum anterior posterior tears, and impingement. A single fellowship-trained physician specializing in shoulder surgery performed all surgeries. In all cases, a diagnostic shoulder arthroscopy was performed using the standard posterior portal in the beach chair position. The rotator interval was identified, and a standard anterior portal was made using an outside-in technique. The biceps tendon was evaluated for irritation, inflammation, tears, instability, and pulley lesions. A probe was placed through the anterior portal to evaluate the superior labrum as well as to pull the LHB tendon into the glenohumeral joint in order to evaluate its mobility and to identify any structural lesions. An open subpectoral biceps tenodesis was performed on all patients with pathologic lesions of the biceps tendon visualized during arthroscopy.

Data for this study were collected retrospectively using the operative report dictated by the surgeon and the MRI report, which had been collected in a computer database. Biceps pathology was classified using the following grading scheme: a normal biceps tendon was one without fraying or other evidence of trauma; a partial tear was a tendon with evidence of fraying or tearing which was not full-thickness, including a longitudinal split tear; a complete tear was defined as discontinuity of the LHB.

\section{Results}

Arthroscopic evaluation of the LHB found 7/66 (10.6\%) of the biceps tendons evaluated to be normal, 43/66 (65.2\%) to have partial tears, fraying, or longitudinal splitting, and 16/66 (24.2\%) to be complete ruptures. Arthroscopic examination also revealed that 64/66 (97\%) patients had associated rotator cuff tears. MRI identified 37 patients as having an intact biceps tendon, of which 23 were normal and 14 were reported as intact but characterized by tendinopathy, degenerative changes, or tenosynovitis. MRI identified 19 patients as having partial LHB tears, and ten patients were found to have complete rupture of the LHB (Table 1). 
Table I Results of biceps pathology as detected by noncontrast MRI compared with direct visualization by arthroscopy

\begin{tabular}{|c|c|c|c|c|c|}
\hline Patient & Age & MRI & Arthroscopy & $\begin{array}{l}\text { Time between } \\
\text { MRI and } \\
\text { arthroscopy } \\
\text { (months) }\end{array}$ & $\begin{array}{l}\text { Associated } \\
\text { rotator } \\
\text { cuff tear }\end{array}$ \\
\hline I & 48 & Normal & Total rupture with retraction & 1.9 & Yes \\
\hline 2 & 52 & Fraying, split, or partial rupture & Fraying, split, or partial rupture & 0.6 & Yes \\
\hline 3 & 49 & Tendinopathy/degenerative/tenosynovitis & Fraying, split, or partial rupture & I.I & No \\
\hline 4 & 55 & Total rupture with retraction & Fraying, split, or partial rupture & 0.7 & Yes \\
\hline 5 & 59 & Tendinopathy/degenerative/tenosynovitis & Fraying, split, or partial rupture & I & Yes \\
\hline 6 & 62 & Fraying, split, or partial rupture & Fraying, split, or partial rupture & 0.9 & Yes \\
\hline 7 & 48 & Fraying, split, or partial rupture & Fraying, split, or partial rupture & I & Yes \\
\hline 8 & 63 & Total rupture with retraction & Total rupture with retraction & $\mathrm{I} .4$ & Yes \\
\hline 9 & 61 & Fraying, split, or partial rupture & Total rupture with retraction & 1.6 & Yes \\
\hline 10 & 50 & Fraying, split, or partial rupture & Fraying, split, or partial rupture & 1.2 & Yes \\
\hline II & 61 & Normal & Fraying, split, or partial rupture & 1.6 & Yes \\
\hline 12 & 52 & Fraying, split, or partial rupture & Fraying, split, or partial rupture & 1.3 & Yes \\
\hline 13 & 66 & Total rupture with retraction & Total rupture with retraction & 0.7 & Yes \\
\hline 14 & 43 & Fraying, split, or partial rupture & Total rupture with retraction & 1.2 & Yes \\
\hline 15 & 67 & Tendinopathy/degenerative/tenosynovitis & Fraying, split, or partial rupture & I.I & Yes \\
\hline 16 & 60 & Total rupture with retraction & Total rupture with retraction & 1.6 & Yes \\
\hline 17 & 53 & Tendinopathy/degenerative/tenosynovitis & Fraying, split, or partial rupture & 2.1 & Yes \\
\hline 18 & 60 & Normal & Fraying, split, or partial rupture & 1.8 & Yes \\
\hline 19 & 44 & Normal & Normal & 1.5 & Yes \\
\hline 20 & 66 & Tendinopathy/degenerative/tenosynovitis & Fraying, split, or partial rupture & 2.3 & Yes \\
\hline 21 & 68 & Total rupture with retraction & Total rupture with retraction & 0.9 & Yes \\
\hline 22 & 55 & Normal & Normal & 3 & Yes \\
\hline 23 & 46 & Fraying, split, or partial rupture & Fraying, split, or partial rupture & 1.2 & Yes \\
\hline 24 & 58 & Tendinopathy/degenerative/tenosynovitis & Fraying, split, or partial rupture & 3.9 & Yes \\
\hline 25 & 61 & Normal & Fraying, split, or partial rupture & 0.4 & Yes \\
\hline 26 & 69 & Normal & Fraying, split, or partial rupture & 0.7 & Yes \\
\hline 27 & 65 & Fraying, split, or partial rupture & Fraying, split, or partial rupture & 3 & Yes \\
\hline 28 & 55 & Normal & Fraying, split, or partial rupture & 0.5 & Yes \\
\hline 29 & 52 & Normal & Fraying, split, or partial rupture & 1.7 & Yes \\
\hline 30 & 62 & Fraying, split, or partial rupture & Fraying, split, or partial rupture & 1.9 & Yes \\
\hline 31 & 60 & Total rupture with retraction & Total rupture with retraction & 2.2 & Yes \\
\hline 32 & 57 & Fraying, split, or partial rupture & Total rupture with retraction & 1.5 & Yes \\
\hline 33 & 62 & Fraying, split, or partial rupture & Fraying, split, or partial rupture & 3.2 & Yes \\
\hline 34 & 64 & Tendinopathy/degenerative/tenosynovitis & Total rupture with retraction & 0.6 & Yes \\
\hline 35 & 65 & Normal & Fraying, split, or partial rupture & 2 & Yes \\
\hline 36 & 61 & Normal & Fraying, split, or partial rupture & 1.7 & Yes \\
\hline 37 & 52 & Fraying, split, or partial rupture & Total rupture with retraction & 0.7 & Yes \\
\hline 38 & 51 & Normal & Fraying, split, or partial rupture & 2.6 & No \\
\hline 39 & 63 & Tendinopathy/degenerative/tenosynovitis & Fraying, split, or partial rupture & 0.6 & Yes \\
\hline 40 & 68 & Normal & Fraying, split, or partial rupture & 0.5 & Yes \\
\hline 41 & 44 & Tendinopathy/degenerative/tenosynovitis & Fraying, split, or partial rupture & 1.1 & Yes \\
\hline 42 & 48 & Fraying, split, or partial rupture & Normal & 0.7 & Yes \\
\hline 43 & 60 & Tendinopathy/degenerative/tenosynovitis & Fraying, split, or partial rupture & 3.5 & Yes \\
\hline 44 & 62 & Normal & Fraying, split, or partial rupture & 0.9 & Yes \\
\hline 45 & 46 & Tendinopathy/degenerative/tenosynovitis & Fraying, split, or partial rupture & 0.7 & Yes \\
\hline 46 & 64 & Fraying, split, or partial rupture & Fraying, split, or partial rupture & 2.3 & Yes \\
\hline 47 & 66 & Fraying, split, or partial rupture & Fraying, split, or partial rupture & 1.5 & Yes \\
\hline 48 & 62 & Tendinopathy/degenerative/tenosynovitis & Fraying, split, or partial rupture & I.I & Yes \\
\hline 49 & 55 & Normal & Fraying, split, or partial rupture & 2.9 & Yes \\
\hline 50 & 65 & Fraying, split, or partial rupture & Total rupture with retraction & 0 & Yes \\
\hline 51 & 45 & Normal & Fraying, split, or partial rupture & 3.4 & Yes \\
\hline 52 & 44 & Total rupture with retraction & Total rupture with retraction & 1.1 & Yes \\
\hline 53 & 61 & Total rupture with retraction & Total rupture with retraction & 1.5 & Yes \\
\hline 54 & 61 & Fraying, split, or partial rupture & Fraying, split, or partial rupture & I.I & Yes \\
\hline
\end{tabular}


Table I (Continued)

\begin{tabular}{|c|c|c|c|c|c|}
\hline Patient & Age & MRI & Arthroscopy & $\begin{array}{l}\text { Time between } \\
\text { MRI and } \\
\text { arthroscopy } \\
\text { (months) }\end{array}$ & $\begin{array}{l}\text { Associated } \\
\text { rotator } \\
\text { cuff tear }\end{array}$ \\
\hline 55 & 56 & Fraying, split, or partial rupture & Fraying, split, or partial rupture & 1.4 & Yes \\
\hline 56 & 63 & Total rupture with retraction & Total rupture with retraction & 0.8 & Yes \\
\hline 57 & 58 & Tendinopathy/degenerative/tenosynovitis & Fraying, split, or partial rupture & 2.8 & Yes \\
\hline 58 & 59 & Normal & Fraying, split, or partial rupture & 0.5 & Yes \\
\hline 59 & 64 & Tendinopathy/degenerative/tenosynovitis & Fraying, split, or partial rupture & 0.8 & Yes \\
\hline 60 & 68 & Total rupture with retraction & Total rupture with retraction & 2.1 & Yes \\
\hline 61 & 43 & Normal & Fraying, split, or partial rupture & 2.8 & Yes \\
\hline 62 & 43 & Normal & Normal & 2.2 & Yes \\
\hline 63 & 66 & Normal & Fraying, split, or partial rupture & 1.6 & Yes \\
\hline 64 & 57 & Normal & Normal & 2.5 & Yes \\
\hline 65 & 62 & Normal & Normal & 1.5 & Yes \\
\hline 66 & 52 & Normal & Normal & 2.1 & Yes \\
\hline
\end{tabular}

Abbreviation: MRI, magnetic resonance imaging.

Direct visualization by diagnostic arthroscopy identified $59 / 66(89.4 \%)$ of patients as having loss of biceps integrity (partial tearing, intra-articular split, or complete rupture). Equivalent findings in biceps pathology were recognized on both MRI and arthroscopic images in 23/66 of the patients studied, for a concordance value of $34.9 \%$. MRI identified only 18 of the 43 partial LHB tendon tears that were visualized during arthroscopy (Figure 1A and B).

The sensitivity, specificity, PPV, and NPV were calculated based on the final data for each possible outcome: normal, partial LHB tear, and complete LHB tear. For a normal LHB tendon in the setting of anterior shoulder pain, noncontrast MRI had a sensitivity of $85.7 \%$, a specificity of $70.0 \%$, a PPV of $25.0 \%$, and a NPV of $97.7 \%$. For a partial LHB tear, noncontrast MRI had a sensitivity of $27.7 \%$, a specificity of $84.2 \%$, a PPV of $81.2 \%$, and a NPV of $32.0 \%$. For a complete LHB tear, noncontrast MRI had a sensitivity of $56.3 \%$, a specificity of $98.0 \%$, a PPV of $90.0 \%$, and a NPV of $87.5 \%$.

\section{Discussion}

In order to evaluate the accuracy of noncontrast MRI for the diagnosis of partial and complete lesions of the LHB, we conducted a retrospective review of 66 patients who underwent shoulder arthroscopy for pain that was at least partially attributed to pathology of the LHB based on physical examination and MRI findings. Using arthroscopic examination as the gold standard, the sensitivity, specificity, PPV, and NPV of noncontrast MRI for the detection of both partial and complete LHB lesions were found to be poor. This is the first study to critically evaluate the accuracy of noncontrast MRI for the detection of LHB pathology.
Noncontrast shoulder MRI is often performed to evaluate the shoulder in preparation for possible surgical intervention, and in many cases the information gained from reading an MRI may help the surgeon to plan for surgery. For instance, MRI without the use of intravenous or intra-articular contrast agents has been found to be accurate for the detection of rotator cuff pathology. Pathologic lesions of the LHB have been given more attention in recent years, as the role of the biceps in pain generation is now better understood. In many cases, pathologic changes of the LHB tendon are found in conjunction with rotator cuff tears and subacromial impingement. ${ }^{13,14}$ Unfortunately, the results of our study indicate that many surgeons will encounter biceps pathology at surgery that was not obvious based on preoperative MRI, and which may even be missed by many musculoskeletal radiologists.

MRI images are produced using energy emitted by tissues and liquids (fat, muscle, tendon, edema) after stimulation of their protons by radiofrequency in the presence of a magnetic field. ${ }^{15}$ Multiple radiofrequency pulses are repeated at specific intervals, within a specific anatomic region of interest, while the magnetic field is varied to excite protons. The excitation of protons and detection of emitted energy create signals that are transformed into signal intensities as a function of position in space and are converted to gray scale for interpretation as an image. ${ }^{15}$ For this study, we utilized a 3.0 Tesla (T) MRI device. In contrast to scanners with lower field strengths, such as $1.5 \mathrm{~T}$, the $3.0 \mathrm{~T}$ MRI is characterized by a distinct signal gain that is transferred into a higher spatial resolution and shorter acquisition time. ${ }^{16}$ The stronger magnetic field created by a 3.0 T magnet can yield better anatomic detail when compared to a $1.5 \mathrm{~T}$ magnet. Thickening and inhomogeneity of the tendon with increased 

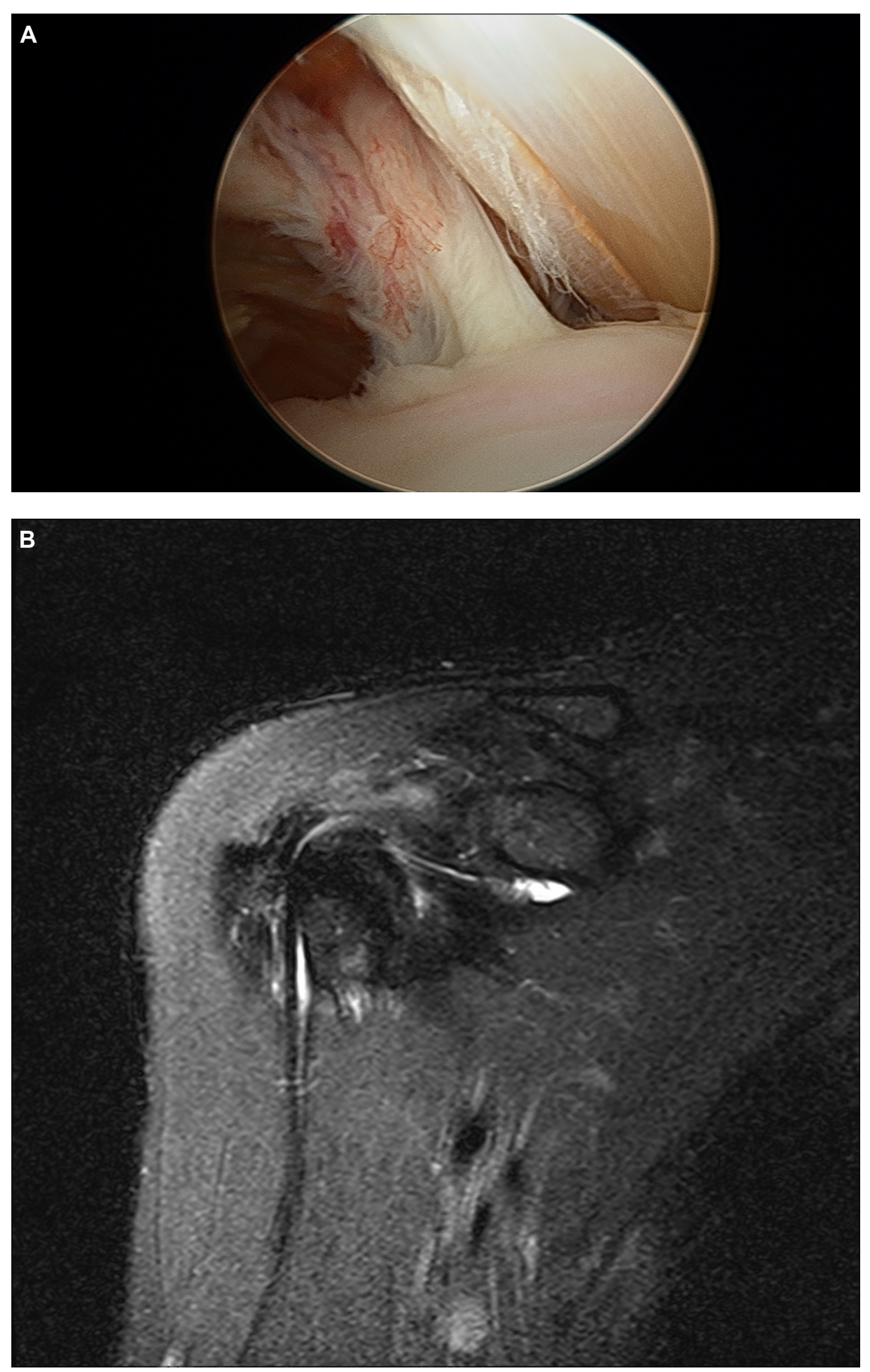

Figure I Arthroscopic image of a partial long head of biceps tendon tear (A), and preoperative coronal T2 MRI of the same patient, with normal biceps pathology reported by radiology $(\mathbf{B})$.

Abbreviation: MRI, magnetic resonance imaging.

signal on all pulse sequences are imaging findings consistent with tendinopathy or tendon degeneration. ${ }^{17}$ Tendinopathy of the LHB is frequently associated with impingement and rotator cuff tears, and in such cases MRI will demonstrate fluid in the joint that extends into the bicipital groove, although this is a nonspecific sign. ${ }^{17}$ Previous studies have concluded that fluid in the tendon sheath of the LHB is abnormal only if it completely surrounds the tendon in the absence of a joint effusion. ${ }^{18}$ In our study, $97 \%$ of patients had rotator cuff tears visualized on MRI and confirmed by arthroscopy, and it is possible that increased fluid within the joint and surrounding the biceps tendon may have led to false MRI interpretations of biceps tendinopathy. Rotator cuff tears may also interfere with the qualitative assessment of the LHB, especially if the 
tears are in close proximity to the biceps pulley, which would lead to some missed diagnoses of partial LHB tears.

Based on our results, standard noncontrast MRI appears to have limited value in detecting pathologic changes of the LHB, which is in agreement with some of the previous studies of the LHB using advanced musculoskeletal imaging. In a prospective study comparing MRI with intravenous gadolinium to arthroscopy in evaluating patients with shoulder pain, Mohtadi et $\mathrm{al}^{7}$ found MRI to have poor correlation with arthroscopic findings in the detection of biceps tendon pathology. The authors noted a concordance of only $37 \%$ between arthroscopic examination and MRI imaging using a 1.5 Tesla magnet with intravenous gadolinium. MRI also misdiagnosed half of the arthroscopically-diagnosed partial tears, which were confirmed arthroscopically in only ten patients. ${ }^{7}$ Ultrasound, which has demonstrated accuracy in detecting full-thickness rotator cuff tears, LHB tendon dislocations, and complete LHB tendon tears has also demonstrated poor accuracy in detecting partial LHB tears. ${ }^{6,9}$ Armstrong et $\mathrm{al}^{6}$ conducted a prospective study comparing ultrasound to arthroscopic findings of biceps pathology and found ultrasound to have a high sensitivity (97\%) in diagnosing normal biceps tendons but a low sensitivity (49\%) for the detection of partial tendon tears or ruptures. The authors concluded that while ultrasound can reliably diagnose complete rupture, subluxation, or dislocation of the biceps tendon, it is not reliable in detecting intra-articular partial thickness tears. ${ }^{6}$

The use of intra-articular gadolinium contrast has been shown to improve the accuracy of MRI for the detection of LHB tears. MRI without the use of intra-articular contrast relies on the interpretation of signal intensities that are not as obviously different as they would be in the case of a magnetic resonance (MR) arthrogram, leading to some instances of false positive readings (case 42, Table I). A study by Zanetti et $\mathrm{al}^{10}$ demonstrated that MR arthrography is sensitive and moderately specific for the diagnosis of LHB tendon pathology, citing a sensitivity of $92 \%$ and a specificity of $56 \%$. The authors suggested that an accurate diagnosis of biceps tendinopathy and rupture may be made if it is assessed in both the axial and parasagittal planes. MR arthrography has also been shown to aid in the detection of associated pathology with tendinitis of the biceps tendon, including labral lesions and rotator cuff tears. ${ }^{8,10}$ Zanetti et $\mathrm{al}^{8}$ concluded that MR arthrography improves the likelihood of properly diagnosing tendinopathy and ruptures of the long biceps tendon as compared to standard MRI, although there were no data given to compare the two imaging modalities. The results of our study support this conclusion. Unfortunately, MR arthrography is an invasive procedure with inherent risks to the patient, and therefore it is not commonly utilized in most institutions. ${ }^{10}$

The limitations of our study relate to its retrospective nature, the lack of healthy control subjects, and the fact that the surgeon performing the arthroscopic examination was not blinded to the findings of the preoperative MRI. However, we feel that our study is an accurate portrayal of the current environment in which a surgeon would be expected to make decisions regarding diagnosis and treatment of LHB pathology. The results of our study emphasize the importance of the physical exam in detecting disorders of the LHB and will allow practicing surgeons to improve their decisionmaking and resource utilization.

\section{Conclusion}

MRI analysis was inaccurate in detecting partial tears and complete ruptures of the LHB tendon. Because standard noncontrast MRI of the shoulder is limited in detecting pathologic lesions of the LHB, it has become our practice to enter the operating room expecting that the diagnosis of LHB pathology will need to be made arthroscopically. We do not routinely use MR arthrography for the detection of LHB tears or other pathology. If no other pathology is detected preoperatively by standard MRI, but the physical examination findings are indicative of LHB pathology, we offer the patient a diagnostic arthroscopy with the option of subpectoral biceps tenodesis if pathologic changes are found.

\section{Disclosure}

This study was not funded by any external source. The authors report no conflicts of interest in this work.

\section{References}

1. Alpantaki K, McLaughlin D, Karagogeos D, Hadjipavlou A, Kontakis G. Sympathetic and sensory neural elements in the tendon of the long head of the biceps. J Bone Joint Surg Am. 2005;87(7):1580-1583.

2. Elser F, Braun S, Dewing CB, Giphart JE, Millett PJ. Anatomy, function, injuries, and treatment of the long head of the biceps brachii tendon. Arthroscopy. 2011;27(4):581-592.

3. Longo UG, Loppini M, Marineo G, Khan WS, Maffulli N, Denaro V. Tendinopathy of the tendon of the long head of the biceps. Sports Med Arthrosc. 2011;19(4):321-332.

4. Nho SJ, Strauss EJ, Lenart BA, et al. Long head of the biceps tendinopathy: diagnosis and management. J Am Acad Orthop Surg. 2010;18(11):645-656.

5. Abboud JA, Bartolozzi AR, Widmer BJ, DeMola PM. Bicipital groove morphology on MRI has no correlation to intra-articular biceps tendon pathology. J Shoulder Elbow Surg. 2010;19(6):790-794.

6. Armstrong A, Teefey SA, Wu T, et al. The efficacy of ultrasound in the diagnosis of long head of the biceps tendon pathology. J Shoulder Elbow Surg. 2006;15(1):7-11. 
7. Mohtadi NG, Vellet AD, Clark ML, et al. A prospective, double-blind comparison of magnetic resonance imaging and arthroscopy in the evaluation of patients presenting with shoulder pain. J Shoulder Elbow Surg. 2004;13(3):258-265.

8. Pfirrmann CW, Zanetti M, Weishaupt D, Gerber C, Hodler J. Subscapularis tendon tears: detection and grading at MR arthrography. Radiology. 1999;213(3):709-714.

9. Teefey SA, Hasan SA, Middleton WD, Patel M, Wright RW, Yamaguchi K. Ultrasonography of the rotator cuff. A comparison of ultrasonographic and arthroscopic findings in one hundred consecutive cases. J Bone Joint Surg Am. 2000;82(4):498-504.

10. Zanetti M, Weishaupt D, Gerber C, Hodler J. Tendinopathy and rupture of the tendon of the long head of the biceps brachii muscle: evaluation with MR arthrography. AJR Am J Roentgenol. 1998;170(6):1557-1561.

11. Neviaser RJ. Lesions of the biceps and tendinitis of the shoulder. Orthop Clin North Am. 1980;11(2):343-348.

12. O’Brien SJ, Pagnani MJ, Fealy S, McGlynn SR, Wilson JB. The active compression test: a new and effective test for diagnosing labral tears and acromioclavicular joint abnormality. Am J Sports Med. 1998; 26(5):610-613.
13. Harrison AK, Flatow EL. Subacromial impingement syndrome. J Am Acad Orthop Surg. 2011;19(11):701-708.

14. Murthi AM, Vosburgh CL, Neviaser TJ. The incidence of pathologic changes of the long head of the biceps tendon. J Shoulder Elbow Surg. 2000;9(5):382-385.

15. Cousins JP, Haughton VM. Magnetic resonance imaging of the spine. $J$ Am Acad Orthop Surg. 2009;17(1):22-30.

16. von Engelhardt LV, Kraft CN, Pennekamp PH, Schild HH, Schmitz A, von Falkenhausen M. The evaluation of articular cartilage lesions of the knee with a 3-Tesla magnet. Arthroscopy. 2007;23(5):496-502.

17. Beltran J, Jbara M, Maimon R. Shoulder: labrum and bicipital tendon. Top Magn Reson Imaging. 2003;14(1):35-49.

18. Kaplan PA, Bryans KC, Davick JP, Otte M, Stinson WW, Dussault RG MR imaging of the normal shoulder: variants and pitfalls. Radiology. 1992;184(2):519-524.

\section{Publish your work in this journal}

Open Access Journal of Sports Medicine is an international, peer-reviewed, open access journal publishing original research, reports, reviews and commentaries on all areas of sports medicine. The manuscript management system is completely online and includes a very quick and fair peer-review system.

\section{Dovepress}

Visit http://www.dovepress.com/testimonials.php to read real quotes from published authors. 